(ㄷ Г.А. Матвеев, Т.И. Голикова, А.А. Васильева, Е.Ю. Васильева, А.Ю. Бабенко*, Е.В. Шляхто

Национальный медицинский исследовательский центр имени В.А. Алмазова, Санкт-Петербург, Россия

Обоснование. Ожирение является мировой неинфекционной пандемией. Низкая эффективность лечения ожирения связана со сложностью удержания потери веса из-за реакции системы регуляции аппетита. В преодолении этой проблемы могут помочь препараты с центральными механизмами действия.

Цель. Сравнение эффектов лираглутида и сибутрамина (Редуксина) на динамику веса и кардиометаболических параметров у пациентов с ожирением без сердечно-сосудистых заболеваний (СС3).

Материалы и методы. Мы оценили динамику основных метаболических параметров (индекс массы тела (ИМТ), глюкоза, показатели липидного обмена, артериальное давление (АД)), уровня гормонов, вовлеченных в регуляцию жирового метаболизма (лептин, адипонектин, инсулин), индекса Homeostasis Model Assessment of Insulin Resistance (HOMA-IR), маркеров оксидативного стресса и воспаления у пациентов с ожирением на терапии лираглутидом или редуксином в течение 6 мес.

Результаты. В соответствии с заявленными критериями включения/исключения в исследование были включены 64 пациента с ожирением: 25 пациентов - в группу «Лираглутид», 39 пациентов - в группу «Сибутрамин».

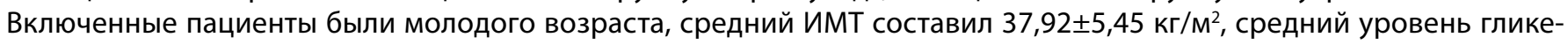

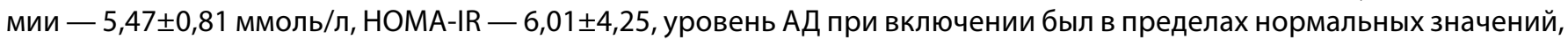
но 21,8\% пациентов получали гипотензивную терапию по поводу артериальной гипертензии (АГ). Оба препарата обеспечили сопоставимое снижение массы тела (-10,28\% vs -9,47\%; p=0,13), уровня лептина $(-32,12 \%$ vs $-41,77 \%$;

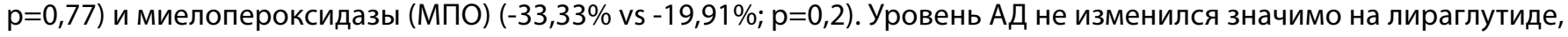
а на редуксине существенно повысился уровень диастолического АД (дАД) (6,87\%; р=0,006). На терапии лираглути-

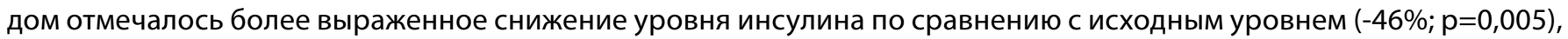
а также уменьшение индекса HOMA-IR $(-50,08 ; p=0,005)$. Повышение уровня адипонектина (+45,36\% vs $14,01 \%$; $\mathrm{p}=0,0045)$ и снижение липопротеидов низкой плотности (ЛПНП) было значительно более выражено на редуксине (-15,03\% vs -9,4\%; p=0,006). В группе «Лираглутид» $36 \%$ участников досрочно завершили участие в исследовании в связи с отсутствием эффекта в виде снижения массы тела. Побочные эффекты в группе «Лираглутид» наблюдались у 16\% больных. 48\% пациентов принимали участие в исследовании в течение 6 мес. В группе «Сибутрамин» 33,4\% пациентов досрочно завершили участие в исследовании по причинам, не связанным с приемом препарата, побочные эффекты отмечались у 20,5\% пациентов. $46,1 \%$ участников группы «Сибутрамин» получали терапию в течение 6 мес.

Заключение. В настоящем исследовании подтверждены ранее полученные данные о том, что терапия как лираглутидом, так и редуксином обеспечивает эффективное снижение массы тела. Мы также обнаружили положительную динамику маркеров воспаления, атерогенеза и оксидативного стресса, уровня лептина. Терапия лираглутидом сопровождалась более выраженным эффектом на состояние углеводного обмена, а терапия редуксином обеспечила более выраженную динамику липидных нарушений и адипонектина. Обе группы характеризовались достаточно низкой приверженностью к терапии, но частота побочных эффектов, потребовавших остановки терапии, была выше в группе «Сибутрамин».

КЛЮЧЕВЫЕ СЛОВА: ожирение; снижение массы тела; биомаркеры; лираглутид; Редуксин.

\title{
COMPARISON OF THE EFFECTS OF LIRAGLUTIDE AND SIBUTRAMINE IN OBESE PATIENTS
}

(c) Georgy A. Matveev, Tatiana I. Golikova, Anastasia A. Vasileva, Elena Yu. Vasilieva, Alina Y. Babenko*, Evgeny V. Shlyakhto

Almazov National Research Centre, St. Petersburg, Russia

BACKGROUND: Obesity is a global noncommunicable pandemic. The low effectiveness of treating obesity is associated with the difficulty of maintaining weight loss due to the reaction of the appetite regulation system. Drugs with central mechanisms of action can help overcome this problem.

AIM: The aim of our study was to compare the effects of liraglutide and sibutramine (Reduxin) on the dynamics of weight and cardiometabolic parameters in obese patients without cardiovascular diseases. 
MATERIALS AND METHODS: We estimated the dynamics of the main metabolic parameters (BMI, glucose, lipid metabolism, blood pressure), the level of hormones involved in the regulation of fat metabolism (leptin, adiponectin, insulin), the HOMA-IR index, markers of oxidative stress and inflammation during therapy with liraglutide in comparison with reduxin for 6 months in obese patients.

RESULTS: 64 obese patients were included in the study: 25 patients — in the "Liraglutide" group, 39 patients — in the "Sibutramine" group in accordance with the declared inclusion / exclusion criteria. The included patients were young, average body mass index (BMI) $(37.92 \pm 5.45 \mathrm{~kg} / \mathrm{m} 2)$, average glycemic level was $5.47 \pm 0.81 \mathrm{mmol} / \mathrm{l}, \mathrm{HOMA}-\mathrm{IR}$ was $6.01 \pm 4.25$, blood pressure was at inclusion was within the normal range, but $21.8 \%$ of patients received antihypertensive therapy.

Both treatment options provided a comparable decrease in body weight $(-10.28 \%$ vs $-9.47 \%, p=0.13)$., Leptin level $(-32.12 \%$ vs $-41.77 \%, p=0.77)$ and myeloperoxidase $(-33.33 \%$ vs $-19.91 \%, p=0.2)$. The blood pressure level did not change significantly on liraglutide, while on reduxin the level of diastolic blood pressure (dBP) increased significantly $(6.87 \%, p=0.006)$. There was a more pronounced decrease in insulin levels compared to the baseline level $(-46 \%, p=0.005)$, as well as a decrease in the HOMA-IR index $(-50.08, p=0.005)$ on liraglutide therapy.An increase in adiponectin levels $(+45.36 \%$ vs $14.01 \%$, $p=0.0045)$ and a decrease in low density lipoprotein(LDL) cholesterol were significantly more pronounced on reduxin therapy $(-15.03 \%$ vs $-9.4 \%, p=0.006)$.

$36 \%$ of the participants completed their participation in the study ahead of schedule due to the lack of effect in the form of weight loss in the "Liraglutide» group. Side effects in the "Liraglutide" group were observed in 16\% of patients. 48\% of patients took part in the study within 6 months. In the «Sibutramine» group 33.4\% of patients completed the study ahead of schedule for reasons unrelated to the drug intake, the side effects were observed in $20.5 \%$ of patients. $46.1 \%$ of participants in the «Sibutramine» group received therapy for 6 months.

CONCLUSIONS: This study confirms the previous findings that both liraglutide and reduxin therapy provide effective weight loss. We found a positive trend in markers of inflammation, atherogenesis and oxidative stress, and leptin levels. Liraglutide therapy was accompanied by a more pronounced effect on the state of carbohydrate metabolism, and reduxin therapy provided a more pronounced dynamics of lipid disorders and adiponexin. Both groups were characterized by a rather low adherence to therapy, but the incidence of side effects requiring stopping therapy was higher in the Sibutramine group.

KEYWORDS: obesity; body weight loss; biomarkers; liraglutide; reduxin.

\section{ОБОСНОВАНИЕ}

Ожирение уже давно стало одним из наиболее распространенных неинфекционных заболеваний, остающимся, несмотря на множество усилий по борьбе с ним, неразрешимой проблемой. Висцеральное ожирение (BO) представляет собой основной фактор риска развития диабета 2 типа (СД2) и ассоциированных с ним других факторов кардиометаболического риска - дислипидемии, артериальной гипертензии (АГ) и, как следствие, сердечно-сосудистых заболеваний (СС3) [1]. Высокие степени ожирения влекут за собой развитие суставной и мышечной патологии, нарушение дыхания и психологические проблемы, которые могут значительно влиять на качество жизни. Бремя перечисленных коморбидных проблем влечет за собой повышение риска смерти.

Ключевые подходы к лечению ожирения направлены на коррекцию причинных факторов и включают изменение характера питания и образа жизни. При постоянном соблюдении рекомендаций эти подходы высокоэффективны и благотворно влияют на прогноз. Даже относительно небольшое снижение веса, на 5\%, может снизить риск развития СС3, СД2, обструктивного апноэ во сне, АГ и послужить катализатором для дальнейшей положительной динамики [2].

Однако лечение ожирения как в мире, так и в Российской Федерации (РФ) является крайне малоэффективным. Основной проблемой является даже не снижение массы тела, добиться которого на фоне интенсивного изменения образа жизни удается менее чем у половины тучных людей, а удержание достигнутого эффекта. По результатам анализа 14 рандомизированных контролируе- мых исследований (РКИ), представленного Mann и соавт., примерно 2/3 пациентов, успешно снизивших вес при изменении образа жизни, в последующие 4-7 лет набирают больший вес, чем имели до вмешательства. Основные причины такого «рикошета» включают индукцию физиологического ответа на снижение веса с повышением продукции орексигенных гормонов и гиперактивность системы «еда-награда» у пациентов с ожирением. Не вызывает сомнений ключевая роль нарушения эффектов орексигенных (грелин и, вероятно, глюкозозависимый инсулинотропный пептид) и анорексигенных гормонов желудочно-кишечного тракта (ЖКТ), включая глюкагоноподобный пептид 1 (ГПП-1), в дисрегуляции этой системы и развитии гиперфагии, характерной для пациентов с ожирением. В связи с этим в реализации подходов к снижению веса в настоящее время акцент переставлен на привлечение тех вариантов вмешательств, которые действуют на центральные механизмы регуляции аппетита, снижая неблагоприятные эффекты этой гормональной перестройки на чувство голода/насыщения. В итоге они позволяют не только эффективно снизить вес, но и удержать достигнутый результат [3].

В РФ на данный момент зарегистрировано два препарата с центральными механизмами действия - это лираглутид и сибутрамин [4]. Определение «ниши» использования для каждого из них является важной клинической задачей.

Сибутрамин избирательно ингибирует пресинаптический обратный захват моноаминергических нейротрансмиттеров (серотонина, норадреналина и, в меньшей степени, дофамина) в центральной нервной системе. Эти нейротрансмиттеры усиливают аппетит, а блокада 
передачи их сигналов - значительно снижает. Сибутрамин также оказывает анорексигенный эффект, уменьшая высвобождение орексигенных нейропептидов в аркуатном ядре. Это обеспечивает ускорение наступления чувства сытости и поддержание его в течение длительного времени. Таким образом, сибутрамин способствует не только активной потере веса, но и, что еще более важно, формирует новый стереотип пищевого поведения [5]. Еще одним важным механизмом его действия является усиление термогенеза путем стимуляции $\beta 2$ - и $\beta 3$-адренорецепторов жировой ткани, что сопровождается активацией процессов липолиза. Результаты рандомизированных клинических исследований (РКИ) подтвердили клинически значимую потерю веса на терапии сибутрамином, но опасения по поводу сердечно-сосудистых (CС) побочных эффектов из-за активации симпатической нервной системы (СНC) ограничивают его использование. Рыночное разрешение на сибутрамин было приостановлено в Италии в марте 2002 г. на основании выявления множественных случаев (50) побочных реакций, в том числе 2 случаев смерти от ССЗ. Спектр побочных эффектов включал тахикардию, гиперемию кожи с чувством жара, мигрень, повышение АД и ухудшение течения гипертонии, тошноту, диспепсию, жажду, сухость во рту, бессонницу, головокружения, нервозность, беспокойство, возбудимость, эмоциональную лабильность, потливость. Европейский комитет по патентованным лекарственным средствам и Управление здравоохранения Великобритании впоследствии провели независимые обзоры эффектов сибутрамина и пришли к выводу, что профиль «риск-польза» остается положительным [6]. Тем не менее были введены ограничения по его назначению для пациентов с СС3, недостаточным контролем АД, рекомендован мониторинг АД и частоты сердечных сокращений в процессе терапии.

Для уменьшения выраженности побочных эффектов был разработан комбинированный препарат Редуксин, содержащий в своем составе сибутрамин и микрокристаллическую целлюлозу. Последняя обладает сорбционными и неспецифическими дезинтоксикационными свойствами, что обеспечивает повышение профиля безопасности препарата. Для лечения ожирения в РФ используется именно этот препарат сибутрамина.

В РФ результаты исследования ПРИМАВЕРА подтвердили отсутствие повышенного риска использования сибутрамина в комбинации с микрокристаллической целлюлозой (Редуксин) в рутинной клинической практике (РКП) у пациентов без клинически значимых СС3, продемонстрировав низкую частоту нежелательных явлений [7].

В последнее время в лечении ожирения с целью нормализации массы тела, пищевых привычек начали использоваться препараты группы агонистов рецепторов глюкагоноподобного пептида-1 (арГПП-1), в частности, лираглутид.

Лираглутид - представитель группы арГПП-1, препаратов, продемонстрировавших уменьшение активности системы «еда-награда» как у животных в эксперименте, так и у людей. Прием данных препаратов сопровождается уменьшением продукции грелина и повышением продукции ГПП-1 [8]. Лираглутид является аналогом ГПП-1 человека длительного действия с 97\% аминокислотной гомологией по отношению к человеческому эндогенному ГПП-1. Рецепторы для ГПП-1 обнаружены в панкреатических альфа- и бета-клетках, центральной и периферической нервной системе, сердце, легких и ЖКТ, что предполагает наличие как центральных, так и периферических эффектов. Лираглутид стимулирует секрецию инсулина, ингибирует секрецию глюкагона и задерживает опорожнение желудка [9].

Прием лираглутида обеспечивает клинически значимую потерю веса до 13,5\% и улучшение кардиометаболических параметров (снижение гликемии, АД и уровня атерогенных липидов). Как пациенты с ожирением без СД, так и пациенты с ожирением и предиабетом имели дополнительное снижение массы тела по сравнению с плацебо (6,2\% против 0,2\%, p<0,0001 и 8,0\% против 2,6\%, p<0,001 соответственно) [3].

Между тем далеко не все пациенты демонстрируют выраженное снижение веса при терапии лираглутидом. По данным РКИ, при лечении лираглутидом пациентов с ожирением с/без СД доля снизивших вес на $5 \%$ и более колеблется в пределах 50-63\%.

Следует отметить, что у лираглутида меньшее количество противопоказаний по сравнению с другими средствами для снижения веса, что делает его более безопасным вариантом для коморбидных пациентов с тяжелой СС-патологией [10]. Более того, для пациентов с сахарным диабетом 2 типа (СД2) и ожирением продемонстрирована способность лираглутида снижать риск СС-событий и смерти [11]. Однако в отношении пациентов без СД данный эффект не доказан.

Однако при приеме лираглутида наблюдается довольно высокая частота побочных эффектов со стороны ЖКТ. Среди желудочно-кишечных симптомов были очень распространены тошнота и диарея $(\geq 1 / 10)$, тогда как рвота, запор, боль в животе и диспепсия были относительно распространенными ( $\geq 1 / 100$ до $<1 / 10)$. Редко возникают анафилактические реакции ( $\geq 1 / 10000$ до <1/1000). Кроме того, были описаны данные о зуде, крапивнице и ангионевротическом отеке на фоне терапии [12].

Таким образом, безопасность диктует определенные показания для персонификации выбора лечения ожирения этими двумя препаратами - пациенты без ССЗ и АГ могут быть кандидатами на терапию редуксином, а для пациентов, имеющих противопоказания к терапии редуксином, в приоритете - лираглутид. Однако необходима тщательная оценка в прямых сравнительных исследованиях эффектов данных препаратов на различные кардиометаболические параметры для улучшения персонализации выбора. Мы постарались реализовать решение этой важной практической задачи в данной статье. Для верификации эффектов препаратов на основные целевые процессы, к которым при ожирении, несомненно, относятся хроническое неинфекционное воспаление и оксидативный стресс (ОС), мы оценили динамику наиболее хорошо изученных биохимических маркеров этих процессов. Помимо классического маркера воспаления С-реактивного белка (СРБ), мы включили в исследование оценку миелопероксидазы (МПО) и параоксоназы-1 (ПО-1). МПО - фермент, синтезируемый лейкоцитами и идентифицированный сегодня как маркер атеросклероза, воспаления и нарушения антиоксидантной защиты. МПО образует мощные прооксиданты, и при развитии как 
инфекционного, так и неинфекционного (при ожирении и метаболическом синдроме (МС)) воспаления развившийся вследствие активации МПО и других ферментов ОС приводит к усилению оксидации липидов. Это вызывает дисбаланс прооксидантов/антиоксидантов и прогрессию атеросклеротических изменений в сосудах. МПО также может способствовать атерогенезу, нейтрализуя антиатерогенное действие липопротеинов высокой плотности (ЛПВП) [13]. Показано, что при определенных условиях МПО, ПО-1 и ЛПВП могут образовывать комплексы, в которых МПО и ПО-1 взаимно ингибируют активность друг друга. МПО считают независимым фактором риска атеросклероза и маркером воспаления [13].

ПО-1 выступает как противовес МПО, являясь универсальным фактором антиоксидантной защиты. Основными ее функциями являются предотвращение окисления липопротеинов, уменьшение образования липидных пероксидов и снижение риска развития атеросклероза. ПО-1 в циркуляции находится в связанном с ЛПВП виде, что обеспечивает ее стабильность, поэтому низкий уровень ЛПВП ассоциирован со снижением ПО-1. Снижение в сыворотке крови активности ПО-1 ассоциировано с усилением OC и повышением риска развития атеросклероза [14], что позволяет рассматривать ее как значимый фактор СС-прогноза. Оценка динамики данных показателей в комплексе с кардиометаболическими и гормональными параметрами может быть полезной для понимания механизмов действия препаратов на снижение веса и факторы СС-риска. В то же время высокий уровень факторов, ассоциированных с ОС, воспалением и высоким риском прогрессии атеросклероза, может быть критерием отбора пациентов для раннего начала медикаментозной терапии ожирения.

\section{ЦЕЛЬ ИССЛЕДОВАНИЯ}

Целью нашего исследования стало сравнение эффектов лираглутида и сибутрамина (Редуксина) на динамику веса и кардиометаболических параметров у пациентов с ожирением без ССЗ.

\section{МАТЕРИАЛЫ И МЕТОДЫ}

Место и время проведения исследования

Место проведения. Исследование проводилось в НМИЦ им. В.А. Алмазова.

Время исследования. Был выполнен анализ данных пациентов с ожирением в период с августа 2017 г. по март 2020 г. в соответствии с критериями включения и невключения. Соответственно продолжительность периода включения составила 2,5 года. Продолжительность периода наблюдения - 6 мес. Сроки контрольных осмотров для определения исходной массы тела и кардиометаболических параметров - 1-14 дней до начала приема препаратов, для оценки динамики массы тела и кардиометаболических параметров - через 3 и 6 мес приема.

Изучаемые популяции (одна или несколько)

Критерии включения: 1) возраст старше 18 лет; 2) ИМТ $>30 \mathrm{Kr} / \mathrm{M}^{2}$.

Критерии исключения: 1) наличие клинически значимой сопутствующей патологии; 2) наличие СД; 3) прием препаратов, влияющих на массу телу (антидепрессанты, нейролептики, аноретики и тому подобное) и уровень гликемии; 4) изменение в режиме приема препаратов для лечения АГ, дислипидемии; 5) хирургическое лечение ожирения в анамнезе; 6) ИМТ >50 кг/M².

Критерии прекращения участия в исследовании: 1) низкая комплаентность пациента; 2) развитие побочных реакций в ходе лечения.

Способ формирования выборки из изучаемой

популяции (или нескольких выборок из нескольких

изучаемых популяций)

Выборка формировалась произвольным способом на основании обследования, включавшего сбор анамнеза, определение антропометрических и лабораторных показателей в соответствии с заданными критериями.

\section{Дизайн исследования}

Неинтервенционное проспективное исследование со сбором архивных данных НМИЦ им. В.А. Алмазова. Для исследования из базы данных были отобраны пациенты, соответствующие критериям включения и исключения.

\section{МЕТОДЫ}

Для исследования из базы данных были отобраны пациенты, соответствующие критериям включения и исключения. До назначения терапии проводили обследование пациентов, включавшее сбор анамнеза, определение антропометрических и лабораторных показателей. Всем пациентам были даны рекомендации по диете (умеренное гипокалорийное питание с формированием дефицита калоража (300-500 ккал), сбалансированное по употреблению углеводов, белков и жиров (углеводы 45-55\%, белки 15-20\%, жиры 20-35\%) с исключением легкоусвояемых углеводов и ограничением животных жиров (не более 10\%) от общего калоража и высоким потреблением грубых волокон (30 г/сут)), режиму питания и дозированным физическим нагрузкам. Определенное ограничение на формирование выборки оказало использование в качестве одного из препаратов сравнения Редуксина. Данный препарат не рекомендуется пациентам с серьезной АГ, ССЗ, что ограничило набор пациентов. Также экономический аспект играл значительную роль в выборе препарата. Пациенты получали терапию Редуксином 10 мг/сут 1 капсула за 30 мин до еды или лираглутидом - стартовая доза 0,6 мг/сут подкожно с увеличением по 0,6 мг с интервалами не менее 1 нед, для улучшения желудочно-кишечной переносимости, до 3 мг/сут подкожно в течение 6 мес соответственно. Для оценки динамики состояния пациента оценка антропометрических данных производилась каждые 3 мес наблюдения, лабораторный контроль показателей - через 6 мес. Произведен сравнительный статистический анализ между группами.

\section{Основной исход исследования}

В качестве основного исхода исследования было принято снижение веса >5\% через 6 мес с учетом данных побочных эффектов.

Для оценки основного исхода использовались антропометрические параметры: масса тела, объем талии (ОТ), объем бедер (ОБ), ИМТ; процент потери массы тела. 
Дополнительные исходы исследования

В качестве дополнительных ожидаемых результатов исследования были приняты динамика кардиометаболических параметров (глюкоза, липиды, артериальное давление); динамика уровня адипокинов (лептин, адипонектин), биохимических маркеров воспаления и оксидативного стресса (СРБ, МПО, ПО-1).

\section{Побочные эффекты лечения; приверженность}

\section{к терапии}

В процессе наблюдения регистрировалось развитие побочных эффектов в группах терапии (со стороны ЖКТ, со стороны СС-системы, СНС, аллергические реакции), сравнивались частота и выраженность побочных эффектов в группах.

Приверженность к терапии оценивалась как процент пациентов, продолживших терапию в течение всего периода наблюдения.

\section{Анализ в подгруппах}

Проводился сравнительный анализ подгрупп пациентов, получавших терапию лираглутидом и Редуксином.

\section{Методы регистрации исходов}

Для исключения пациентов с патологией печени проводился анализ крови на АЛТ, АСТ, общий билирубин, патологией почек - креатинин, скорость клубочковой фильтрации (СКФ; расчет по Кокрофту-Голту), с патологией углеводного обмена - уровень глюкозы плазмы натощак. Исключение патологии со стороны сердечно-сосудистой системы проводилось анамнестически (наличие в анамнезе инфаркта миокарда, инсульта, хронической сердечной недостаточности III-IV ФК (NYHA), АГ с нецелевыми уровнями АД).

Mетоды регистрации антропометрических данных

Всем пациентам выполнялся физикальный осмотр: измерение роста в см, веса в кг, с расчетом ИМТ по формуле: масса тела (кг) / рост² (м), ОТ и ОБ в см и расчетом соотношения ОТ/ОБ, оценивался уровень АД.

\section{Методы регистрачии биохимических}

и метаболических параметров

Оценку биохимических параметров проводили с использованием автоматического анализатора (Cobas c311, Германия) и коммерческих наборов (наборы реагентов, Roshe, Швейцария).

\section{Методы регистрации инсулинорезистентности}

Уровень инсулина в сыворотке крови измеряли с использованием автоматического анализатора (Cobas e411, Roche, Швейцария) и с использованием коммерческих наборов Insulin Elecsys, Cobas, Roche, Швейцария, с диапазоном измерения 1,39-6945 МкME/мл и нормальным значением 17,8-173,0 пмоль/л. Коэффициент пересчета пмоль/л $\times 0,144=$ мкЕ/мл.

Индекс НОМА был рассчитан по формуле для оценки инсулинорезистентности (ИР):

$$
\frac{\text { Глюкоза }\left(\frac{\text { ммоль }}{л}\right) * \text { Инсулин }\left(\frac{\mathrm{MkME}}{\mathrm{Mл}}\right)}{22,5}
$$

Методы регистрации уровня гормонов, вовлеченных в метаболизм жировой ткани

Кроме того, у всех пациентов была выполнена оценка уровня гормонов, вовлеченных в регуляцию метаболизма жиров и углеводов, регуляцию аппетита (лептин, адипонектин) натощак. Уровень адипонектина в плазме крови, а лептина - в сыворотке крови измеряли с помощью иммуноферментного анализа с использованием автоматического анализатора (BioRad 680-монометр, автоматизированный анализатор, США). Для адипонектина (коммерческий набор BioVendor): диапазон измерения 0,026-100 мкг/мл, коэффициент чувствительности 0,026 мкг/мл, для лептина (коммерческий набор для проведения иммуноферментного анализа (ИФА), DBC): диапазон измерения от 2,0 до 11,0 нг/мл, чувствительность 0,5 нг/мл.

\section{Методы регистрации маркеров воспаления}

и оксидативного стресса

Оценку уровня С-реактивного белка (СРБ) проводили с использованием автоматического анализатора (Cobas с311, Германия) и коммерческих наборов (наборы реагентов, Roshe, Швейцария).

Исследование уровня МПО (ИФА) выполнено набором реактивов Human Myeloperoxidase Lot P203356, R\&D Systems, нг/мл, ПО-1 - набором реактивов Paraoxoonase 1 (PON1), Lot L190801668, MyBioSource, мкг/мл.

\section{Статистический анализ}

Принципы расчета размера выборки. Размер выборки предварительно не рассчитывался. Meтoды статистического анализа данных. Статистический анализ был проведен с использованием STATISTICA 10 (StatSoftInc, США) для Windows. Данные были представлены как среднее \pm стандартное отклонение или абсолютное число (\%). Был проведен корреляционный анализ, чтобы установить связь между различными параметрами. Все непараметрические данные были проанализированы с помощью критерия Манна-Уитни. Критический уровень значимости (р) для проверки статистических гипотез при сравнении статистических показателей принимался менее 0,05.

\section{Этическая экспертиза}

Протокол исследования одобрен локальным этическим комитетом НМИЦ им. В.А. Алмазова (протокол № 63 от 14.04.2014). Все пациенты дали письменное информированное согласие на участие в исследовании.

\section{РЕЗУЛЬТАТЫ}

Объекты (участники) исследования

В исследование были включены 64 пациента с ожирением: 25 пациентов были включены в группу «Лираглутид» (терапия лираглутидом 3 мг/сут), 39 пациентов в группу «Сибутрамин» (Редуксин 10 мг/сут), между пациентами двух групп не было выявлено статистически значимых различий. Включенные пациенты были молодого возраста, мужчины составили 31,2\% от группы, средний ИМТ $(37,92 \pm 5,45$ кг/м²) соответствовал 2-й степени ожирения. Средний уровень гликемии был в норме $(5,47 \pm 0,81$ ммоль/л), но у 7,8\% были 
инициальные нарушения углеводного обмена. HOMA-IR

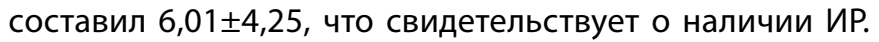
Уровень АД при включении был в пределах нормы $(121,36 \pm 14,17 / 76,0 \pm 6,73$ мм рт. ст.), но 21,8\% пациентов получали гипотензивную терапию по поводу АГ.

\section{Основные результаты исследования}

Уровень адипокинов был изменен типично для пациентов с ожирением - лептин резко повышен, а адипонектин хотя и не отличался от референсных значений, был ниже, чем у здоровых людей [15].

В таблице 1 представлена в процентах динамика основных метаболических параметров (ИМТ, глюкоза, показатели липидного обмена, АД) от начала терапии к 6 мес лечения в группах «Сибутрамин» и «Лираглутид». Из таблицы следует, что оба варианта терапии обеспечили сопоставимое снижение массы тела, однако ОБ в большей степени уменьшился в группе «Сибутрамин» (-8,9\% vs -4,6\%; $\mathrm{p}=0,012)$. По данным липидограммы у пациентов на фоне терапии лираглутидом не обнаружилось значимой динамики уровня липидов (общий холестерин (ОХС), триглицериды (ТГ), липопротеины низкой плотности (ЛПНП)). При этом в группе «Сибутрамин» через 6 мес наблюдалось улучшение липидного профиля в виде снижения ЛПНП $(p=0,006)$. Однако межгрупповые различия не имели статистической значимости. Уровень гликемии по сравнению с исходным не изменился ни в одном из вариантов лечения, хотя имелась отчетливая тенденция к более выраженному снижению в группе лираглутида $(p=0,051)$. Уровень систолического АД (сАД) имел тенденцию к снижению на лираглутиде $(p=0,026)$ и не изменился на Редуксине. В то же время уровень диастолического АД (дАД) существенно повысился на терапии Редуксином $(p=0,006)$ и не изменился на лираглутиде. В итоге имела место тенденция к более выраженному снижению сАД (сибутрамин $+0,65 \%$ vs лираглутид $-9,6 \%, p=0,086)$ на лираглутиде, не достигшая статистической значимости, но по дАД имелись статистически значимые различия с выраженной положительной динамикой на лираглутиде (сибутрамин $+6,81 \%$ vs лираглутид $-7,2 \%, p=0,005)$.

В таблице 1 представлена динамика изученных гормонов в группах терапии. Как видно из представленных данных, на фоне терапии лираглутидом отмечалось более выраженное снижение уровня инсулина по сравнению с исходным уровнем ( $p=0,005)$, а также уменьшение индекса НОМА $(p=0,005)$, в то время как у пациентов группы «Сибутрамин» отмечались только тенденции к снижению уровня инсулина $(p=0,09)$ и индекса НОМА $(p=0,06)$, не достигшие статистической значимости. Уровень лептина значимо снизился в обеих группах ( $p=0,001$ для Редуксина и $\mathrm{p}=0,004$ для лираглутида). В то же время повышение уровня адипонектина было значительно более выражено на Редуксине («Сибутрамин» $+45,36 \%$ vs «Лираглутид» +14,01\%; p=0,0095).

Исходный уровень МПО у включенных в исследование пациентов значимо превышал верхний порог нормы, а уровень ПО-1 был в пределах референсного диапазона. Следует отметить, что уровень МПО значимо прямо коррелировал с ОТ ( $r=0,36 ; p=0,021)$, что свидетельствует о связи ее активности с висцеральным характером ожирения. В процессе терапии как в группе «Лираглутид», так и в группе «Сибутрамин» наблюдалось выраженное снижение уровня МПО (p=0,005). Статистически значимых различий в динамике уровня СРБ зарегистрировано не было в обеих группах. Уровень ПО-1 также значимо не изменился, но имелась тенденция к его повышению, более выраженная на Редуксине. Таким образом, оба препарата оказывали положительное влияние на маркеры атерогенеза и ОС.

\section{Нежелательные явления}

Оба варианта терапии (лираглутид и Редуксин) сопровождались развитием побочных эффектов.

Побочные эффекты в группе «Лираглутид» наблюдались у 12,5\% больных в виде диспептических явлений (диарея, тошнота, рвота, опоясывающие боли в эпигастрии), у 4\% развилась генерализованная аллергическая реакция по типу крапивницы, в связи с чем терапия была прекращена, 9 пациентов (36\%) досрочно завершили участие в исследовании в связи с отсутствием эффекта в виде снижения массы тела.

В итоге только 12 пациентов (48\%) получали терапию лираглутидом в дозе 3 мг/сут в течение 6 мес.

В группе «Сибутрамина» побочные эффекты отмечались у 20,5\% пациентов, из них 5,1\% пациентов завершили прием препарата в связи с развитием побочных эффектов со стороны ЖКТ, у 10,2\% наблюдались побочные эффекты со стороны сердечно-сосудистой системы в виде подъема АД более 140/90 мм рт.ст., появления жалоб на учащенное сердцебиение, у 5,1\% развились побочные эффекты со стороны нервной системы, 33,4\% пациентов завершили участие в исследовании по причинам, не связанным с приемом препарата. Таким образом, частота и выраженность побочных эффектов были большими в группе «Сибутрамина». 18 пациентов $(46,1 \%)$ группы «Сибутрамин» получали терапию в течение 6 мес.

Резюме основного результата исследования

Исследуемые препараты (лираглутид и Редуксин) обеспечили сопоставимое снижение массы тела, сопровождавшееся положительной динамикой маркеров воспаления, атерогенеза и оксидативного стресса, уровня лептина. При этом терапия лираглутидом сопровождалась более выраженным эффектом на состояние углеводного обмена, а терапия редуксином - более выраженной динамикой липидных нарушений и уровня адипонектина.

\section{ОБСУЖДЕНИЕ ОСНОВНОГО РЕЗУЛЬТАТА ИССЛЕДОВАНИЯ}

Репрезентативность выборок

Репрезентативность выборки подтверждается тем, что поло-возрастные характеристики, частота НУО, липидных нарушений, АГ, средний ИМТ сопоставимы с таковыми в большинстве крупных РКИ и исследований РКП.

Небольшой объем выборки объясняется тем, что исследование носило одноцентровой характер и включало пациентов, посещавших НМИЦ им. В.А. Алмазова в ограниченный период времени. Определенное ограничение на формирование выборки оказало использование в качестве одного из препаратов сравнения Редуксина. Данный препарат не рекомендуется пациентам с серьезной АГ, ССЗ, что ограничило набор пациентов. 


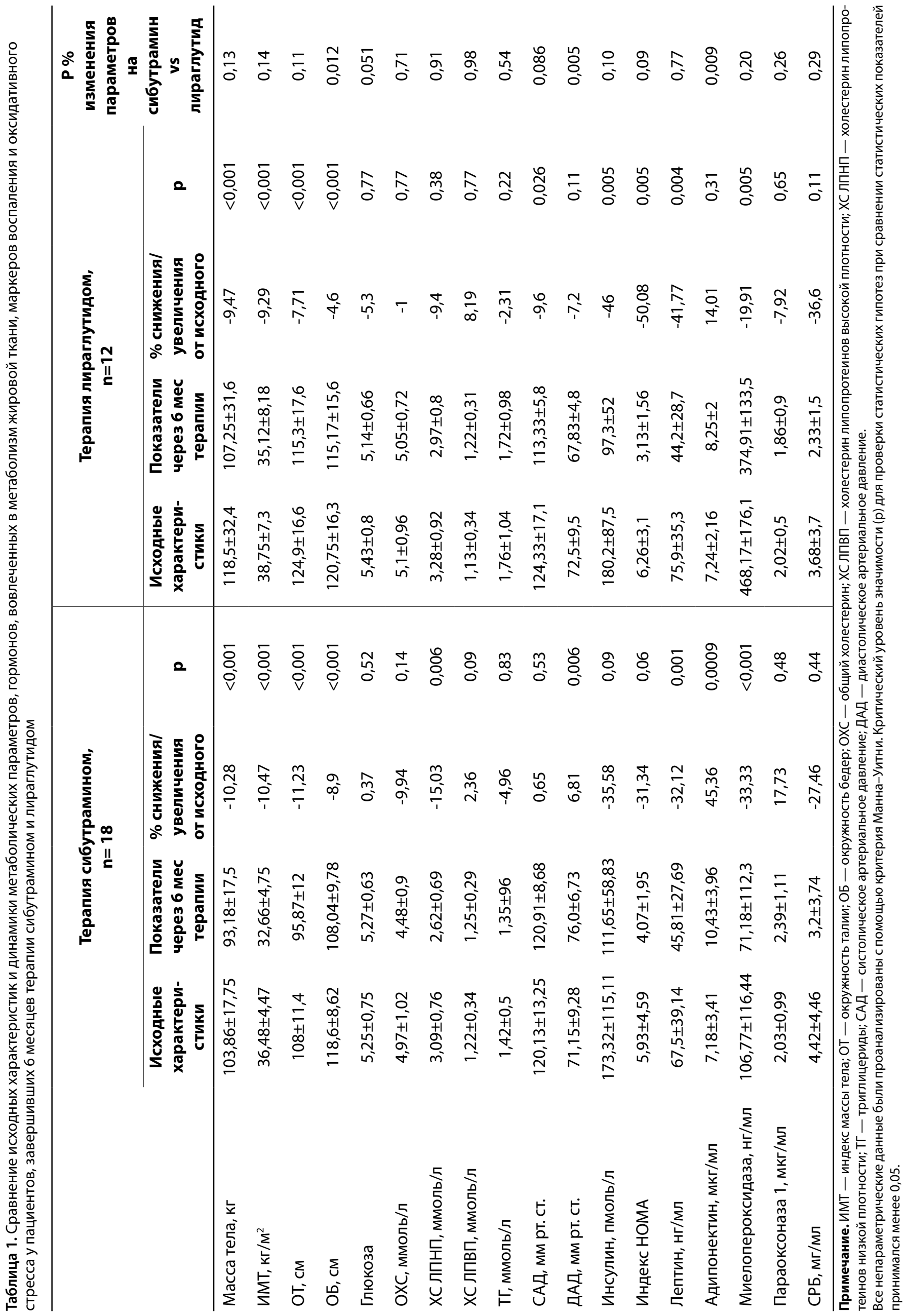


Сопоставление с другими публикациями

Через 6 мес терапии снижение массы тела >5\% отмечено у $91 \%$ пациентов группы «Лираглутида» и у $88 \%$ группы «Сибутрамина». Эти результаты несколько превышают значения, продемонстрированные в РКИ. Так, в исследовании SCALE в группе пациентов с ожирением без СД, участники, хорошо ответившие на терапию изменением образа жизни (потеря >5\% массы тела), были рандомизированы на 2 группы: Лираглутид 3,0 мг или плацебо. В группе, получавшей лираглутид, наблюдалось значительно большее снижение массы тела, чем в группе плацебо (6,2\% против 0,2\%; p <0,0001), а доля пациентов, достигших как минимум 5\% потери веса, составила 50,5\% против 21,8\% в группе плацебо за 56 нед [10]. Аналогичным образом дело обстоит с исследованиями по Редуксину. По данным наблюдательного исследования в РКП ПРИМАВЕРА снижение массы тела через 3, 6 и 12 мес лечения составило 9,5, 15,1 и 19,7\% соответственно. В нашем исследовании процент потери массы тела к 6 месяцу терапии также превысил 10\%. В недавнем метаанализе РКИ продолжительностью не менее одного года (10 исследований с 2623 пациентами) сибутрамин снижал массу тела на 4,2 кг (3,6-4,7 кг) больше, чем плацебо. Более высокая доля пациентов, получавших сибутрамин, достигла как >5\% потери веса (55\% против 27\% в группе плацебо), так и >10\% потери веса (28\% против 10\% в группе плацебо) [16].

Были получены данные о значимом снижении выраженности ИР на лираглутиде, что соответствует данным РКИ, продемонстрировавшим большую эффективность лираглутида в отношении снижения риска развития СД2 у пациентов с ранее выявленными нарушениями углеводного обмена (НУO). По данным исследования SCALE, на 160-й неделе только у 1,8\% пациентов, получавших лираглутид 3,0 мг/сут, развился СД2 по сравнению с 6,2\% пациентов в группе плацебо. Риск развития СД2 был снижен на 79\% в группе пациентов, получавших терапию лираглутидом [10]. Тот факт, что в нашем наблюдении не отмечено значимого снижения уровня глюкозы, может объясняться как существенно меньшим периодом наблюдения, так и малым размером выборки. Кроме того, в крупном метаанализе [16] отмечено, что наибольшую динамику уровня глюкозы имеют пациенты с НУО, которых в нашем исследовании было немного (8\%), в отличие от исследования SCALE, где это было критерием включения. Это же объяснение может быть дано к результатам по Редуксину.

В исследовании «ВЕСНА» среди пациентов с исходно имеющимся СД2 и другими НУО было отмечено снижение показателей глюкозы венозной крови на 7\%. В исследовании ПРИМАВЕРА, где большинство участников не имело НУО (СД2 был у 18,8\%, НТГ - у 3,3\%, суммарно $22,2 \%)$ не представлены данные о снижении риска СД2 на фоне терапии Редуксином. В отличие от результатов ПРИМАВЕРА, демонстрировавших улучшение метаболических параметров, в частности, снижение уровня АД (сАД на 2,36 и дАД 2,09 мм рт. ст.; $\mathrm{p}<0,001$ ) на фоне снижения массы тела, нами отмечено отсутствие динамики сАД и некоторое повышение дАД. Между тем в исследовании ПРИМАВЕРА, если во время двух последовательных визитов отмечались повышение сАД и/или дАД на 10 мм рт. ст. или отмечался уровень АД выше 145/90 мм рт. ст., паци- енту рекомендовалась отмена препарата. Соответственно, пациенты с тенденцией к повышению АД выбывали из исследования [17]. В нашем исследовании пациенты исходно были нормотензивны, поэтому даже повышение дАД на 4,6 мм рт. ст. не приводило к превышению значения 90 мм рт. ст., и они продолжали участие в исследовании. В целом, несмотря на выявленное повышение дАД, декомпенсации АГ не происходило, и это не требовало отмены терапии.

На обоих препаратах отмечалась низкая приверженность к терапии (в группе «Лираглутид» - 48\%, в группе «Сибутрамин» - 46,1\%). Это значительно ниже, чем по данным РКИ лираглутида (75\% участников завершили участие в исследовании). Это, вероятно, обусловлено тем, что одной из основных причин отказа от терапии лираглутидом в РКП является высокая стоимость препарата. В некоторых исследованиях имеются данные о более низкой приверженности на инъекционных препаратах, в частности арГПП-1. К сожалению, сопоставить наши результаты с другими исследованиями РКП мы не смогли, так как в представленных в литературе исследованиях лираглутида не оценивалась приверженность к терапии.

Приверженность к терапии на Редуксине была сопоставима с группой «Лираглутида» и существенно ниже, чем в РКИ. Учитывая, что стоимость препарата не столь высока, как для лираглутида, а форма приема препарата (per os, один раз в день) комфортна, можно предполагать, что снижение приверженности в этой группе определяется ухудшением качества жизни из-за побочных эффектов. По данным РКП (ПРИМАВЕРА), в течение 6 мес Редуксин принимали 59,3\% пациентов [18], что несколько выше, чем в нашем исследовании. К сожалению, анализ причин прекращения терапии в этом исследовании не представлен, кроме указания на то, что побочные эффекты отмечались у 4,1\%, и только в $0,18 \%$ случаев это стало основанием для прекращения терапии. По результатам РКИ, 21,4\% не завершили исследование.

Развитие побочных эффектов наблюдалось у $16,5 \%$ участников группы Лираглутид и несколько чаще (20,6\%) - группы «Сибутрамин». Наиболее частыми побочными эффектами являлись нарушения со стороны ЖКТ в виде диспептических явлений (12,5\%), в 4\% случаев развилась генерализованная аллергическая реакция по типу крапивницы. По данным РКИ [12], диспепсия встречалась с сопоставимой частотой, а анафилактические реакции, данные о зуде, крапивнице описаны крайне редко на фоне терапии лираглутидом $(\geq 1 / 10000$ до <1/1000). Таким образом, полученные нами данные по побочным эффектам, сопоставимы с данными РКИ.

Спектр побочных эффектов Редуксина был представлен нарушениями со стороны ЖКТ (5,1 \% случаев), сердечно-сосудистой системы (10,5\% случаев), нервной системы (5,1 \% случаев), что обусловлено присущими ему симпатомиметическими свойствами. Как уже отмечено, по данным исследования РКП ПРИМАВЕРА Редуксин продемонстрировал хорошую переносимость, и общее количество пациентов с нежелательными явлениями составило 4,1\%. Не было зарегистрировано серьезных побочных эффектов, связанных с приемом препарата [18]. Частота побочных эффектов у включенных в наше исследование пациентов была значительно выше - 20,5\%. В то же время в РКИ, сравнившем две схемы приема 
сибутрамина (непрерывный и прерывистый) в течение 44 нед, частота побочных эффектов, обычно вызываемых сибутрамином, составила 17\% при непрерывном лечении и $14 \%$ - при прерывистой терапии. Эти данные сопоставимы с нашими результатами.

\section{Клиническая значимость результатов}

Полученные данные свидетельствуют о сопоставимой эффективности терапии лираглутидом и Редуксином в отношении снижения веса в процессе 6-месячной терапии. В то же время терапия лираглутидом сопровождалась более выраженным эффектом на выраженность ИР в виде значительного снижения уровня инсулина

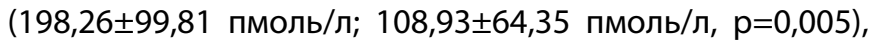
а также уменьшения индекса $\operatorname{HOMA}(7,39 \pm 5,19 ; 3,58 \pm 2,22$; $\mathrm{p}=0,005)$, хотя снижение уровня глюкозы не достигло статистической значимости.

Оба препарата обеспечивали значимое снижение уровня лептина. Данный эффект описан во многих исследованиях, изучавших влияние снижения веса на динамику адипоцитокинов. В то же время Редуксин продемонстрировал значимо более выраженное повышение уровня адипонектина при сопоставимом снижении веса, что может отражать особенности его механизма действия. Известно, что выраженное снижение противовоспалительного гормона адипонектина отмечается у пациентов с ВО [19], и его повышение может быть ассоциировано с улучшением кардиоваскулярного прогноза. Учитывая выраженные антиатерогенные свойства адипонектина, можно предположить, что повышение этого гормона содружественно с уменьшением активности МПО обеспечило статистически значимое снижение уровня ЛПнП.

Мы обнаружили существенное повышение уровня МПО при ожирении до начала терапии, степень которого была взаимосвязана с ОТ, а уровень ПО-1 был в норме. Между тем, по данным литературы, активность ПО-1 у пациентов с ожирением может быть как нормальной, так и сниженной. В недавнем исследовании Cervellati C. и соавт [20] была проанализирована связь активности ПО-1 со степенью ожирения и было показано, что активность ПО-1 снижена только у пациентов с морбидным ожирением (ИМТ \40 кг/м2). В процессе терапии как лираглутидом, так и сибутрамином наблюдалось выраженное снижение уровня МПО, а уровни СРБ и ПО-1 не имели существенной динамики, но имелась тенденция к повышению ПО-1, более выраженная на Редуксине. Таким образом, оба препарата оказывали положительное влияние на маркеры ОС и воспаления. При анализе литературы мы не обнаружили публикаций, в которых бы анализировались эффекты изученных нами препаратов на уровень МПО и ПО-1, что позволяет полагать наши данные новыми и представляющими определенный интерес с точки зрения уточнения механизмов действия данных препаратов на сердечнососудистый риск у пациентов с ожирением.

\section{Направления дальнейших исследований}

В ходе работы мы идентифицировали ряд предикторов ответа на терапию каждым из изученных препаратов. В плане персонификации подходов к выбору медикаментозной терапии ожирения нам представляется полезным сравнить эффективность данных препаратов при их назначении на основании наличия предикторов хорошего ответа. Такой подход может обеспечить как более высокую эффективность, так и лучшую переносимость и приверженность к терапии.

\section{Ограничения исследования}

К ограничениям исследования можно отнести довольно небольшой объем выборки, что, в частности, связано с довольно низкой приверженностью к приему не обеспечиваемых по льготе препаратов в реальной клинической практике при длительных периодах наблюдения. Существенным ограничением исследования является отсутствие рандомизации на прием того или иного препарата, что связано с наличием ограничений по приему сибутрамина у пациентов с сопутствующей кардиопатологией (высокие уровни АД, ишемическая болезнь сердца и т.п.). Это, в свою очередь, ограничило когорту пациентов достаточно молодыми людьми без существенной сердечно-сосудистой патологии.

\section{ЗАКЛЮЧЕНИЕ}

Экономический аспект играет значительную роль в обеспечении высокой приверженности, а соответственно, и эффективности медикаментозной терапии ожирения. Для снижения неэффективных расходов важно отбирать на лечение каждым препаратом пациентов, которые получат наибольшую пользу от лечения именно этим препаратом.

Полученные в настоящем исследовании данные позволяют утверждать, что терапия Редуксином может быть эффективным вмешательством у пациентов с ожирением 1-2-й степени и низким риском развития СД. Учитывая более значимую динамику ОБ на терапии Редуксином, можно предполагать, что глютеофеморальный тип ожирения является опцией выбора для терапии сибутрамином, тем более что при этом варианте ожирения реже встречаются противопоказания к нему.

Терапия лираглутидом, по данным наших более ранних исследований, более эффективна у пациентов с ИМТ от 35 до 45 кг/м² и обеспечивает более выраженный эффект на НУО, а потому особо показана пациентам с дисгликемией. Учитывая высокую стоимость препарата, отбор кандидатов на лечение лираглутидом должен проводиться с использованием шкал предикции ответа во избежание неэффективных финансовых потерь. Это тем более важно, что одним из наиболее сильных предикторов высокой приверженности к терапии лираглутидом, по данным как наших [3], так и зарубежных [21] исследований, является процент потери веса на ранних сроках терапии (3 мес). Хорошие результаты вскоре после старта вмешательства создают позитивный настрой и повышают желание пациента продолжить терапию.

\section{ДОПОЛНИТЕЛЬНАЯ ИНФОРМАЦИЯ}

Источники финансирования. Исследование выполнено на средства, выделенные для выполнения государственного задания № 2021-24 в НМИЦ им. В.А. Алмазова.

Конфликт интересов: Авторы декларируют отсутствие явных и потенциальных конфликтов интересов, связанных с содержанием настоящей статьи 
Участие авторов. Матвеев Г.А. - получение и анализ данных (набор пациентов группы сибутрамина, сбор анамнеза, объективный осмотр, обследование), статистический анализ данных, написание раздела статистический анализ, формирование таблиц, частичное написание раздела результаты исследования (по пациентам, получавшим сибутрамин); Голикова Т.И. - получение и анализ данных (набор пациентов группы лираглутида, сбор анамнеза, объективный осмотр, обследование), подбор литературы для раздела актуальность, его написание, частичное написание раздела результаты исследования (по пациентам, получавшим лираглутид), написание аннотации и ее перевод; Васильева А.А. - получение и анализ данных (формирование базы данных пациентов), описание раздела объекты исследования в статье; Васильева Е.Ю. - получение и анализ данных (получение и оценка лабораторных результатов), описание методик лабораторных исследований, написание раздела методы обследова- ния; Бабенко А.Ю. - концепция и разработка дизайна исследования, интерпретация результатов, написание разделов дизайн исследования, обоснование, формирование цели исследования, резюме результата исследование, обсуждение результата исследования, общая коррекция рукописи с целью повышения научной ценности статьи; Шляхто Е.В. - концепция и разработка дизайна исследования, внесение в рукопись существенной (важной) правки с целью повышения научной ценности статьи. Все авторы одобрили финальную версию статьи перед публикацией, выразили согласие нести ответственность за все аспекты работы, подразумевающую надлежащее изучение и решение вопросов, связанных с точностью или добросовестностью любой части работы.

Благодарности. Авторы выражают благодарность Мосикян А.А., Деревицкому И.В. за помощь в разработке дизайна и статистическом анализе.

\section{СПИСОК ЛИТЕРАТУРЫ | REFERENCES}

1. Colditz GA, Willett WC, Rotnitzky A, et al. Weight gain as a risk factor for clinical diabetes mellitus in women. Ann Intern Med. 1995;122(7):481-486. doi: https://doi.org/10.7326/0003-4819-122-7-199504010

2. Sharon MF. Obesity: Risk factors, complications, and strategies for sustainable long-term weight management. J Am Assoc Nurse Pract. 2017;29(1):3-14. doi: https://doi.org/10.1002/2327-6924.12510

3. Тихоненко Е.В., Бабенко А.Ю., Шляхто Е.В. Предикторы эффективности терапии агонистами рецепторов глюкагоноподобного пептида-1 у пациентов с сахарным диабетом 2 типа и ожирением // Ожирение и метаболизм. 2018. - T. 15. — №4. - C. 22-30. [Tikhonenko EV, Babenko AY, Shlyakhto EV. Predictors of effectiveness of glucagon-like peptide-1 receptor agonist therapy in patients with type 2 diabetes and obesity. Obesity and metabolism. 2018;15(4):22-30. (In Russ.)]. doi: $10.14341 /$ omet 9584

4. Диагностика, лечение, профилактика ожирения и ассочиированных с ним заболеваний. Начиональные клинические рекомендачии / Под ред. Шляхто Е.В. Санкт-Петербург; 2017. [Diagnostika, lecheniye, profilaktikaozhireniyaiassotsiirovannykhsnimzabolevaniy. Natsional'nyye klinicheskiye rekomendatsii. Ed. by Shlyakhto EV. Saint-Petersburg; 2007. (In Russ.)].

5. Smith I, Goulder M. Randomized placebo-controlled trial of longterm treatment with sibutramine in mild to moderate obesity. J Fam Pract. 2001;50:505-512.

6. The European Agency for the Evaluation of Medicinal Products Post-authorisation Evaluation of Medicines for Human Use. Committee for proprietary medicinal products opinion following an article 31 referral sibutramine. 2 December 2002 CPMP/4514/02/ Final. Available from: https://www.ema.europa.eu/en/committees/ committee-medicinal-products-human-use-chmp

7. Дедов И.И., Мельниченко Г.А., Романцова Т.И. Стратегия управления ожирением: итоги Всероссийской наблюдательной программы «ПримаВера» // Ожирение и метаболизм. 2016. - T. 13. — №1. - C. 36-44. [Dedov II, Melnichenko GA, Romantsova TI. The strategy of obesity management: the results of All-Russian observational program «Primavera». Obesity and metabolism. 2016;13(1):36-44. (In Russ.)]. doi: https://doi.org/10.14341/OMET2016136-44

8. Wadden TA, Volger S, Sarwer DB, et al. A Two-Year Randomized Trial of Obesity Treatment in Primary Care Practice. N Engl J Med. 2011;365:1969-1979. doi: https://doi.org/10.1056/NEJMoa1109220

9. Madsbad S. Liraglutide Effect and Action in Diabetes (LEAD ${ }^{\mathrm{TM}}$ ) trial. Expert Rev Endocrinol Metab. 2009;4(2):119-129. doi: https://doi.org/10.1586/17446651.4.2.119

10. Mehta A, Marso SP, Neeland IJ. Liraglutide for weight management: a critical review of the evidence. Obes Sci Pract. 2017;3(1):3-14. doi: https://doi.org/10.1002/osp4.84 IF 3.969

11. Kumbhani DJ. Liraglutide effect and action in diabetes: evaluation of cardiovascular outcome results - leader. American college of cardiology; 2018.
12. Filippatos TD, Panagiotopoulou TV, Elisaf MS. Adverse Effects of GLP-1 Receptor Agonists. Rev Diabet Stud. 2014;1 1(3-4):202-230. doi: https://doi.org/10.1900/RDS.2014.11.202

13. Гавриленко Т.И, Рыжкова Н.А., Пархоменко О.М. Миелопероксидаза и ее роль в развитии ишемической болезни сердца // Український кардіологічний журнал. 2014. — №4. - C. 119-126. [Gavrylenko TI, Ryzhkova NO, Parkhomenko OM. Myeloperoxidase and its role in development of ischemic heart disease. Ukrainian cardiology journal. 2014:4:119-126 (In Russ.)].

14. Rosenblat M, Volkova N, Ward J, et al. Paraoxonase 1 (pon1) inhibits monocyte-to-macrophage differentiation. Atherosclerosis. 2011;219(1):49-56. doi: https://doi.org/10.1016/j.atherosclerosis.2011.06.054

15. Бабенко А.Ю., Матвеев Г.А., Алексеенко Т.И., и др. Взаимосвязи компонентов метаболического синдрома с уровнем гормонов, вовлеченных в регуляцию метаболизма жировой ткани // Артериальная гипертензия. - 2019. - Т. 25. №6. - C. 639-652. [Babenko AY, Matveev GA, Alekseenko TI, et al. Interrelations of components of metabolic syndrome with the level of the hormones involved in regulation of adipose tissue metabolism. Arterial Hypertension. 2019;25(6):639-652. (In Russ.)]. doi: https://doi.org/10.18705/1607-419X-2019-25-6-639-65216.

16. Rucker D, Padwal R, Li SK, et al. Long term pharmacotherapy for obesity and overweight: updated meta-analysis. BMJ. 2007;335(7631):1194-1199. doi: https://doi.org/10.1136/bmj.39385.413113.25

17. Weeke P, Andersson C, Fosbøl EL, et al. The Weight Lowering Effect of Sibutramine and Its Impact on Serum Lipids in Cardiovascular High Risk Patients With and Without Type 2 Diabetes Mellitus An Analysis From the SCOUT Lead-In Period. BMC Endocr Disord. 2010;26;10:3. doi: https://doi.org/10.1186/1472-6823-10-3

18. Dedov II, Melnichenko GA, Troshina EA, et al. Body Weight Reduction Associated with the Sibutramine Treatment: Overall Results of the PRIMAVERA Primary Health Care Trial. Obes Facts. 2018;1 (4):335-343. doi: https://doi.org/10.1159/00048888019.

19. Кологривова И.В., Винницкая И.В., Кошельская О.А., Суслова Т.Е. Висцеральное ожирение и кардиометаболический риск: особенности гормональной и иммунной регуляции // Ожирение и метаболизм. - 2017. - Т. 14. - №3. - 3-10. [Kologrivova IV, Vinnitskaya IV, Koshelskaya OA, Suslova TE. Visceral obesity and cardiometabolic risk: features of hormonal and immune regulation. Obesity and metabolism. 2017;14(3):3-10. (In Russ.)]. doi: https://doi.org/10.14341/OMET201733-1

20. Cervellati C, Bonaccorsi G, Trentini A, et al. Paraoxonase, arylesterase and lactonase activities of paraoxonase-1 (PON1) in obese and severely obese women. Scand J Clin Lab Invest. 2018;78(1-2):18-24. doi: https://doi.org/10.1080/00365513.2017.1405274

21. Cuomo A, Bolognesi S, Goracci A, et al. Feasibility, Adherence and Efficacy of Liraglutide Treatment in a Sample of Individuals With Mood Disorders and Obesity. FrontPsychiatry. 2018;9:784. doi: https://doi.org/10.3389/fpsyt.2018.00784 


\section{ИНФОРМАЦИЯ ОБ АВТОРАХ [AUTHORS INFO]:}

*Бабенко Алина Юрьевна, д.м.н. [Alina Y. Babenko, MD, PhD]; адрес: Россия, 197341, Санкт-Петербург, ул. Аккуратова, д. 2 [address: 2 Akkuratova street, 197341 Saint-Petersburg, Russia]; ORCID: https://orcid.org/0000-0002-0559-697X; eLibrary SPIN: 9388-1077; Researcher ID: Q-6714-2016; e-mail:alina_babenko@mail.ru

Матвеев Георгий Александрович, аспирант [Georgy A. Matveev, MD, postgraduate student]; ORCID: https://orcid.org/0000-0002-2695-4924; e-mail: zx5000@mail.ru

Голикова Татьяна Игоревна, аспирант [Tatiana I. Golikova, MD, postgraduate student]; ORCID: https://orcid.org/0000-0003-4376-3721; eLibrary SPIN: 2527-5440; e-mail: tanya.golya@gmail.com

Васильева Анастасия Алексеевна, аспирант [Anastasia A. Vasileva, MD, postgraduate student]; ORCID: https://orcid.org/0000-0003-0919-7607: e-mail: dr.volynets@gmail.com

Васильева Елена Юрьевна [ElenaYu. Vasilieva, MD]; ORCID: https://orcid.org/0000-0003-3185-3957; eLibrary SPIN: 8546-5546; e-mail: elena-almazlab@yandex.ru

Шляхто Евгений Владимирович, д.М.Н., професcop [Evgeny V. Shlyakhto, MD, PhD, Professor]; ORCID:https://orcid.org/0000-0003-2929-0980; eLibrary SPIN: 6679-7621; e-mail: e.shlyakhto@almazovcentre.ru

*Автор, ответственный за переписку / Corresponding author.

\section{ЦИТИРОВАТЬ:}

Матвеев Г.А., Голикова Т.И., Васильева А.А., Васильева Е.Ю., Бабенко А.Ю., Шляхто Е.В. Сравнение эффектов терапии ожирения лираглутидом и сибутрамином // Ожирение и метаболизм. - 2021. — Т. 18. — №2. — С. $218-228$. doi: https://doi.org/10.14341/omet12498

\section{TO CITE THIS ARTICLE:}

Georgy A. Matveev, Tatiana I. Golikova, Anastasia A. Vasileva, Elena Yu. Vasilieva, Alina Y. Babenko, Evgeny V. Shlyakhto. Comparison of the effects of liraglutide and sibutramine in obese patients. Obesity and metabolism. 2021;18(2):218-228. doi: https://doi.org/10.14341/omet12498 